\title{
An automatic precipitation-phase distinction algorithm for optical disdrometer data over the global ocean
}

\author{
Jörg Burdanowitz ${ }^{1}$, Christian Klepp ${ }^{2}$, and Stephan Bakan ${ }^{1}$ \\ ${ }^{1}$ Max Planck Institute for Meteorology, Bundesstraße 53, Hamburg, Germany \\ ${ }^{2}$ University of Hamburg, CliSAP/CEN, Bundesstraße 55, Hamburg, Germany \\ Correspondence to: Jörg Burdanowitz (joerg.burdanowitz@mpimet.mpg.de)
}

Received: 27 November 2015 - Published in Atmos. Meas. Tech. Discuss.: 21 December 2015

Revised: 10 March 2016 - Accepted: 28 March 2016 - Published: 13 April 2016

\begin{abstract}
The lack of high-quality in situ surface precipitation data over the global ocean so far limits the capability to validate satellite precipitation retrievals. The first systematic ship-based surface precipitation data set OceanRAIN (Ocean Rainfall And Ice-phase precipitation measurement Network) aims at providing a comprehensive statistical basis of in situ precipitation reference data from optical disdrometers at $1 \mathrm{~min}$ resolution deployed on various research vessels (RVs). Deriving the precipitation rate for rain and snow requires a priori knowledge of the precipitation phase (PP). Therefore, we present an automatic PP distinction algorithm using available data based on more than 4 years of atmospheric measurements onboard RV Polarstern that covers all climatic regions of the Atlantic Ocean. A time-consuming manual PP distinction within the OceanRAIN post-processing serves as reference, mainly based on 3-hourly present weather information from a human observer. For automation, we find that the combination of air temperature, relative humidity, and 99th percentile of the particle diameter predicts best the PP with respect to the manually determined PP. Excluding mixed phase, this variable combination reaches an accuracy of $91 \%$ when compared to the manually determined PP for $149635 \mathrm{~min}$ of precipitation from RV Polarstern. Including mixed phase (165 $632 \mathrm{~min})$, an accuracy of $81.2 \%$ is reached for two independent PP distributions with a slight snow overprediction bias of 0.93 . Using two independent PP distributions represents a new method that outperforms the conventional method of using only one PP distribution to statistically derive the PP. The new statistical automatic PP distinction method considerably speeds up the data post-processing within OceanRAIN while introducing an objective PP probability for each PP at $1 \mathrm{~min}$ resolution.
\end{abstract}

\section{Introduction}

Rare and often low-quality gauge-based surface reference data sets challenge the in situ validation of oceanic precipitation as observed by passive and active microwave satellite sensors (Taylor, 2000; Adler et al., 2012). Over land, radar and gauge-based precipitation monitoring networks cover a large fraction of the land surface for more than 2 decades, which qualifies them to validate precipitation satellite estimates (Schneider et al., 2014). However, the ocean surface lacks dense long-term in situ precipitation monitoring networks. Furthermore, existing coastal and island-based precipitation measurements may not fully represent oceanic precipitation because the measured particle size distributions (PSDs), precipitation rates, and accumulations may differ from those measured over the open ocean (Kidd and Levizzani, 2011). However, Bumke and Seltmann (2012) found no difference between PSDs over coastal areas and ocean. Most existing in situ oceanic precipitation data sets sample measurements from low-quality rain gauges on voluntary observing ships (VOSs; Kent et al., 2010) or buoy arrays (Weller et al., 2008). Many of these in situ ocean data sets include present weather observations but lack quantitative estimates of precipitation. The high-latitude ocean completely lacks precipitation measurements that sample solid and mixed-phase precipitation. However, recent and future precipitation satellite estimates demand high-quality in situ quantitative precipitation estimates including snowfall over the global ocean.

The large uncertainty in precipitation gauge measurements arises from the rough open-ocean conditions that complicate precipitation monitoring. Under high wind speed, standard 
rain gauges with horizontal catchment surfaces face a large undercatch (Yuter and Parker, 2001; Michelson, 2004). In the extratropics, mixed-phase and solid precipitation cause further difficulties strongly adding to the undercatch (Goodison, 1978) of horizontally oriented measuring surfaces. In contrast, optical instruments with a vertically oriented measuring surface such as disdrometers perform better at capturing precipitation under high wind speeds, though varying wind directions are challenging. Optical disdrometers are thus denoted as the reference in situ instrument to measure precipitation (Taylor, 2000).

To provide systematic high-quality in situ precipitation data over the ocean, the long-term Ocean Rainfall And Ice-phase precipitation measurement Network (OceanRAIN; Klepp, 2015) applies automatic optical disdrometers of type ODM470 that are deployed onboard sea-going research vessels (RVs) for operation in all climatic regions. The ODM470 was developed to measure under high wind speed and frequently varying wind directions. Its cylindrical measuring volume ensures being independent from the wind-driven incidence angle of the falling hydrometeors while a wind vane keeps the measuring volume perpendicular to the instantaneous wind direction. The ODM470 accuracy lies within a range of $3 \%$ rain accumulation limited to rainfall at various wind conditions with respect to an improved ship rain gauge including side collectors on RV Alkor on the Baltic Sea (Bumke and Seltmann, 2012). Compared to an ANS410 WMO-reference rain gauge over land (Lanza and Vuerich, 2009), the ODM470 deviates by $2 \%$ under low wind speed (Klepp, 2015). For snow, a predecessor of the current ODM470 agreed with the observer's log during the Lofoten Cyclones campaign (LOFZY; Klepp et al., 2010) in detecting snowfall events. More recent results for measuring snow and mixed-phase precipitation are expected soon from the WMO Solid Precipitation InterComparison Experiment (SPICE) at Marshall field site in Boulder (CO, USA), where the ODM470 was compared against a multitude of in situ solid precipitation instruments for more than 2 years. The ODM470 suits well to measure various types of precipitation under open-ocean conditions onboard sea-going RVs.

The deployment of the ODM470 on several RVs allows to sample OceanRAIN precipitation data from all climate zones including the cold-season high latitudes. This requires a precipitation-phase (PP) distinction between rain, snow, and mixed phase in order to provide correct precipitation rates for disdrometer-measured PSDs. The PP information usually originates from human observers' reports saved in the WMO present weather code $w w$ (WMO, 2015). Efforts to automatize present weather observations impose high requirements on instruments such as present weather sensors. Automated present weather sensors encounter problems at temperatures around $0{ }^{\circ} \mathrm{C}$ as well as for light precipitation and small particle sizes (Merenti-Välimäki et al., 2001). High wind speed also complicates the PP determination because the wind speed strongly interferes with the particle fall speed that solely carries the PP information. Thus, most studies to distinguish PPs limit the wind conditions to low wind speed or calm conditions (Löffler-Mang and Joss, 2000; Yuter et al., 2006; Ishizaka et al., 2013). Only few studies apply more sophisticated instruments that use articulating particle size velocity (PARSIVEL) disdrometers to account for wind effects and thus directly derive the PP from the particle fall speed (Friedrich et al., 2013). More simply constructed instruments such as the ODM470 require ancillary data to determine the PP.

In OceanRAIN, we aim to replace the so far manual PP distinction method by an automatic algorithm for three main reasons. First, the manual method consumes a considerable amount of time and workforce because the $1 \mathrm{~min}$ precipitation data requires visual inspection of air temperature, present weather observations, and theoretical rain and snow rate. Second, the human-based PP decision based on visual data inspection lacks objectivity while the decision itself remains non-transparent to the user. Third, temporal gaps exist between the 3-hourly present weather observational timesteps, especially during nighttime adding to the uncertainty. Currently, no measures of this PP uncertainty are provided in the manual method. For these reasons, we present a new automatic PP distinction algorithm including a PP probability for OceanRAIN precipitation data that is also applicable to all other instruments sampling PSDs of precipitation.

The new PP distinction algorithm follows a statistical approach guided by many other studies that relate atmospheric predictors to the PP (Koistinen and Saltikoff, 1998; Fuchs et al., 2001; Dai, 2008; Froidurot et al., 2014). Most previous work focuses on PP distinction over land only, while we introduce a new PP distinction algorithm over the ocean. Dai (2008) compares ocean and land areas using a relatively coarse time step of few to several hours depending on availability of observations. In contrast, OceanRAIN offers atmospheric measurements at $1 \mathrm{~min}$ resolution while present weather observations are limited to 3-hourly timesteps during daytime only. These high-resolution ancillary data from the RV combined with PSD data from the optical disdrometer enable a more accurate and reliable PP distinction.

Section 2 introduces the optical disdrometer, the manual PP distinction method, and the OceanRAIN data set in detail. Section 3 presents different atmospheric variable combinations and methods to predict the PP. In Sect. 3.1, one PP distribution distinguishes between two PPs, while in Sect. 3.2 one PP distribution distinguishes between three PPs. Section 3.3 introduces a newly developed method to predict three PPs using two PP distributions. Section 4 discusses the results by comparing with similar studies. Section 5 completes our investigations with a summary and concluding remarks. 


\section{Data and methods}

Since 2010, OceanRAIN collects atmospheric data including precipitation rates on several RVs. Current permanent deployments include the German ships RV Polarstern (since June 2010), RV Meteor (since March 2014), RV Sonne (since November 2014), and the Russian ship Akademik Ioffe (since September 2010). The backbone of OceanRAIN is the optical disdrometer ODM470, which is explained in detail in Sect. 2.1. Section 2.2 introduces the manual method that has been used so far to retrieve the PP in OceanRAIN. These manually determined PPs function as a benchmarking reference for the new automatic PP distinction algorithm. For the algorithm development, we exclusively use RV Polarstern data (Sect. 2.3) that contains a high fraction of highlatitude solid and mixed-phase precipitation being a prerequisite to develop a robust PP distinction algorithm. While Klepp (2015) describes the OceanRAIN data post-processing and quality checking before PP distinction we focus on presenting a new automatic PP estimation method that provides uncertainty information.

\subsection{The ODM470}

The ODM470 is an optical disdrometer to measure precipitation, manufactured by the German company Eigenbrodt GmbH \& Co KG near Hamburg (Germany). The instrument consists of an infrared (IR) light-emitting diode (LED) at $880 \mathrm{~nm}$ and a photo diode receiver (Lempio et al., 2007). The IR-LED of the ODM470 is only activated once at least 8 particles per minute pass the active sensing area of the precipitation detector IRSS88 (Fig. 1, right) in order to increase IR-LED lifetime and exclude measurement artifacts caused by birds or other non-precipitation objects. The IRSS 88 switches off the ODM470 after 1 min without any particle passing the IRSS 88 active sensing area. The entire ODM470 system was developed in a way to minimize undesired influences by changing wind directions and high wind speed. The ODM470 sensitive optical volume has a cylindrical shape of $120 \mathrm{~mm}$ length and $22 \mathrm{~mm}$ in diameter. The cylindrical shape guarantees an independence from the incidence angle of the falling hydrometeors, which becomes crucial under high wind speeds and superstructure-induced local turbulence. Mounted on a pivotable axis, a wind vane ensures the optical volume to adjust perpendicular to the instantaneous local wind direction. The ODM470 mounting height typically ranges between 30 and $45 \mathrm{~m}$ height, depending on the RV's specifications (Fig. 1). This elevated deployment reduces influences on the measured precipitation by splashing wave water.

During precipitation events, the falling hydrometeors attenuate the emitted IR radiation, which decreases the voltage signal measured. The duration of the voltage drop determines the particle transit time, that is, the total time it takes a particle to pass through the optical volume of the disdrometer.

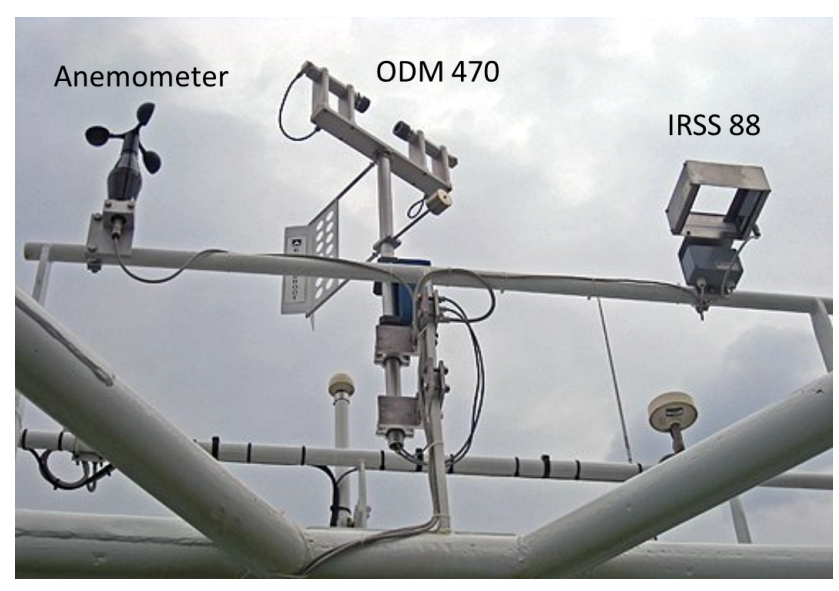

Figure 1. The image displays the automatic ODM470 measurement system including a cup anemometer, the optical disdrometer ODM470, and the precipitation detector IRSS88, deployed in the highest main mast at about $43 \mathrm{~m}$ height onboard RV Polarstern.

From the amplitude of the detector voltage drop the crosssectional area can be deduced, which determines the particle diameter. The measured particle diameters are split into 128 logarithmically distributed size bins, of which the smallest is less than $0.02 \mathrm{~mm}$ and the largest corresponds to the optical volume diameter of $22 \mathrm{~mm}$. However, wind- or waveinduced ship vibrations passed on to the instrument might cause artificial signals that are not distinguishable from real precipitation, which is why particles below bin $14(0.43 \mathrm{~mm}$ diameter) are not considered in OceanRAIN. This exclusion of small particles also removes sea spray particles from the PSD spectra. The remaining particles are accumulated and binned over $1 \mathrm{~min}$. From the resulting PSD, the precipitation rate PR $\left(\mathrm{mm} \mathrm{h}^{-1}\right)$ or liquid water equivalent $\left(\mathrm{kg} \mathrm{m}^{-2} \mathrm{~h}^{-1}\right)$ after Großklaus (1996) can be calculated using

$\mathrm{PR}=3600 \cdot \sum_{\text {bin }=1}^{128} n($ bin $) \cdot v($ bin $) \cdot m($ bin $)$,

where $v$ (bin) $\left(\mathrm{m} \mathrm{s}^{-1}\right)$ denotes the particle terminal fall speed and $m$ (bin) (kg) the particle mass; both are parameterized. $n$ (bin) $\left(\mathrm{m}^{-3}\right)$ denotes the PSD density per bin class that is calculated following Clemens (2002) by considering the geometrical features, diameter $d(\mathrm{~m})$ and length $l(\mathrm{~m})$, the sampling time $t$ (s) of the ODM470 as well as the sum of local wind speed $U_{\text {rel }}\left(\mathrm{m} \mathrm{s}^{-1}\right)$ relative to the ship movement measured by a cup anemometer, and the empirical terminal fall speed $v$ (bin) $\left(\mathrm{m} \mathrm{s}^{-1}\right)$ as

$n($ bin $)=\frac{N(\text { bin })}{l \cdot d \cdot t \cdot \sqrt{U_{\mathrm{rel}}^{2}+[v(\text { bin })]^{2}}}$.

$N$ (bin) is the number of measured particles per bin class, denoted as PSD. As explained, in Eq. (1) empirical relationships utilize the particle diameter $D$ that strongly depends 
on the type of precipitation. Henceforth, we refer to precipitation phase (PP), which means either liquid precipitation (rain), solid precipitation (e.g., snow or graupel), or mixedphase precipitation. For rain the drop mass $m_{1}(\mathrm{~kg})$, or liquid water content, and the particle terminal fall speed $v_{1}\left(\mathrm{~m} \mathrm{~s}^{-1}\right)$ are well known and calculated using Eqs. (3) and (4) from Atlas and Ulbrich (1974), respectively.

$m_{1}=1000 \cdot \frac{4}{3} \pi \cdot(0.5 D)^{3}$

$v_{1}=9.65-10.3 \cdot e^{-600 D}$

For snow, the measured cross-sectional area differs from the required maximum dimension of the particle due to the nonspherical shape of snowflakes. This difference requires applying a transfer function. However, Lempio et al. (2007) found that the product of particle terminal fall speed and particle mass (liquid water equivalent) as a function of crosssectional area is in the same order of magnitude for various frozen precipitation particle types. Hence, no transfer function between cross-sectional area and maximum diameter is required when using a spherical lump graupel assumption. The lump graupel assumption works well for frozen precipitation particles between 0.4 and $9 \mathrm{~mm}$ in diameter, whereas particles exceeding $9 \mathrm{~mm}$ in diameter rarely occur. Nevertheless, events with large particles introduce larger errors to the estimate in the same way as the retrieval quality may largely differ for individual snowfall events. Overall, no unique snowfall retrieval can be derived using optical disdrometers without recording the individual particle shape. Compared to a Geonor gauge, the optical disdrometer agreed well in most cases and overestimated a few light snowfall cases during the 1999/2000 winter period at Uppsala (Lempio et al., 2007). Following the lump graupel approximation by Hogan (1994), particle mass $m_{\mathrm{s}}$ (Eq. 5) and particle terminal fall speed $v_{\mathrm{s}}$ (Eq. 6) are calculated empirically as

$m_{\mathrm{S}}=1.07 \cdot 10^{-5} \cdot(100 D)^{3.1}$,

$v_{\mathrm{s}}=7.33 \cdot(100 D)^{0.78}$.

Klepp et al. (2010) observed lump graupel being the most frequently occurring precipitation type over the cold-season Norwegian Sea during the LOFZY campaign. Battaglia et al. (2010) discuss several sources of error for a snow-measuring PARSIVEL whereof those for particle shape and orientation, margin effects, and coinciding particles also apply to the ODM470. However, the PARSIVEL is more sensitive to influences by wind speed and wind direction on the falling precipitation particles because the PARSIVEL has a fixed non-pivotable horizontal optical sensing area.

For mixed-phase precipitation, we generally use the snow retrieval (Eqs. 5 and 6) to calculate the precipitation rate within OceanRAIN because the absolute error of treating rain drops like snow particles, and thus underestimating the precipitation rate, results in a smaller error than vice versa.
In more than $90 \%$ of the precipitating cases from RV Polarstern in OceanRAIN the precipitation rate calculated with Eqs. (3) and (4) (theoretical rain rate) exceeds precipitation rate calculated with Eqs. (5) and (6) (theoretical snow rate) by a factor of 50 to 200. Accordingly, this large difference might cause large biases in the precipitation rate for misclassified PP. Correctly classified mixed-phase precipitation events might still strongly underestimate the precipitation rate if the instantaneous rain fraction sharply exceeds 0.5. The minute-aggregated fraction of liquid and solid particles cannot be identified by the ODM470 and would require ancillary data such as a video disdrometer. More details on the instrumentation can be found in Lempio et al. (2007) while algorithm features are explained in Klepp (2015).

\subsection{The manual PP distinction}

Though time-consuming, the manual PP distinction was so far employed to determine the PP that is required to calculate the precipitation rate. Because we apply this manual PP distinction data as reference to the new automatic PP distinction algorithm, a detailed explanation follows. If available, shipboard present weather observations stored in the WMO standard meteorological present and past weather code $w w$ (WMO, 2015) are translated into the three PPs: rain, snow, and mixed phase according to Petty (1995), displayed in Table 1 . However, the translation of $w w$ codes into a PP partly differs between OceanRAIN and Petty (1995). While Petty (1995) assigns one single PP to each of the $w w$ codes, OceanRAIN allows multiple PPs for a single ambiguous $w w$ code (bold weather codes in Table 1). Instead, Petty (1995) lists ambiguous $w w$ codes in a category called "indeterminable" (abbreviated "Indet." in Table 1) that, however, includes no PP information anymore. For that reason we deviate from this procedure to retain as much PP information as possible. Another difference concerns $w w$ codes for all kinds of freezing rain (i.e., rain at freezing temperatures) without snow that Petty (1995) classifies as mixed phase. Classifying freezing rain as mixed phase by applying the lump graupel assumption (Eqs. 5 and 6) leads to an underestimated precipitation rate. This underestimation arises because falling raindrops freeze only after passing the disdrometer's optical volume when hitting any obstacle, which is why we consider freezing rain cases in OceanRAIN as rain $(w w=56,57,66,67)$. Likewise, we assign a snow flag to ice pellets $(w w=79)$ as well as mostly to hail ( $w w=89,90)$, graupel $(w w=87,88)$, and combinations of both $(w w=93,94,96,99)$. The aim is mainly to separate frozen (solid) from non-frozen (liquid) precipitation particles to account for differences in density and cross-sectional area that affect Eqs. (3) to (6) and hence the precipitation rate. In contrast, the study by Froidurot et al. (2014) concentrates exclusively on clear rain, snow, and mixed-phase observations (Table 1) by neglecting drizzle, freezing rain, and ice pellets, among others. In general, assigning the correct PP for a given ambiguous $w w$ code re- 
Table 1. Translation of WMO present weather codes $w w$ (WMO, 2015) into the three PPs from Petty (1995), Froidurot et al. (2014), and OceanRAIN. $w w$ codes printed in bold can be translated into multiple PPs in OceanRAIN depending on ancillary data. "Indet./hail" denotes indeterminate precipitation or hail used for classification in Petty (1995).

\begin{tabular}{|c|c|c|c|c|}
\hline Source & Rain & Snow & Mixed phase & Indet./hail \\
\hline Petty (1995) & $\begin{array}{l}21,25,50-55 \\
58-65, \quad 80-82, \\
91-92\end{array}$ & $\begin{array}{l}22,26,70-78 \\
85-86\end{array}$ & $\begin{array}{l}23-24,56-57 \\
66-69,79,83- \\
84\end{array}$ & $\begin{array}{l}20,27-29,87- \\
90,93-99\end{array}$ \\
\hline OceanRAIN & $\begin{array}{l}\mathbf{2 0}, 21,25, \mathbf{2 9} \\
50-67, \quad 80-82, \\
91-92, \mathbf{9 5}, \mathbf{9 7}\end{array}$ & $\begin{array}{l}\mathbf{2 0}, 22, \mathbf{2 6 - 2 7} \\
\mathbf{2 9}, 70-79,85- \\
86, \mathbf{8 7 - 9 0 , 9 3 -} \\
\mathbf{9 5}, 96, \mathbf{9 7}, 99\end{array}$ & $\begin{array}{l}23-24, \mathbf{2 6 - 2 7} \\
\mathbf{2 9}, 68-69,83- \\
84,87-90,93- \\
\mathbf{9 5}, \mathbf{9 7}\end{array}$ & - \\
\hline Froidurot et al. (2014) & $\begin{array}{l}58-65, \quad 80-82 \\
91-92\end{array}$ & $70-79,85-86$ & $68-69,83-84$ & - \\
\hline
\end{tabular}

quires visual inspection of PSDs and ancillary data collected onboard the RV.

The $w w$ code from shipboard observations on RV Polarstern is available 3 hourly during daytime only. Nighttime conditions and PP changes between two consecutive 3hourly observational time steps require ancillary data from the RV to derive the PP. By ancillary data we refer to atmospheric variables measured onboard the ship including the ODM470, such as air temperature, humidity, and precipitation rate. These ancillary data are available at a much higher resolution of $1 \mathrm{~min}$ compared to the 3-hourly observations. Initially, we assign the PP derived from the $w w$ code directly to every single minute of precipitation that follows a 3-hourly observation as a first-guess information. If available, air temperature as one of the ancillary data serves to possibly correct this first-guess PP. For near-freezing air temperatures, the manual procedure calculates the precipitation rate after Eq. (1) for rain (Eqs. 3 and 4) and snow (Eqs. 5 and 6) assumption separately. Large differences between theoretical rain and snow rate can help to identify a plausible PP. However, if both theoretical rain and snow rate differ by much less than 2 orders of magnitude, their influence on the PP decision becomes negligible, which makes the PP more arbitrary. Accordingly, the manual method might be biased by the worker who decides for a PP and the observer on the RV. For these reasons, we aim at developing an automatic PP distinction algorithm at $1 \mathrm{~min}$ resolution that statistically derives a PP from atmospheric measurements.

\subsection{OceanRAIN data from RV Polarstern}

The manual PP estimation has been applied to more than 4 years of OceanRAIN data from RV Polarstern (11 June 2010-8 October 2014). This period consists of several expeditions to Arctic and Antarctic regions. In addition to the high latitudes, RV Polarstern collected precipitation data from the tropics and subtropics when crossing the equator in the Atlantic Ocean six times (Fig. 2). The whole measuring period amounts to more than 268000 min of precipitation ex-

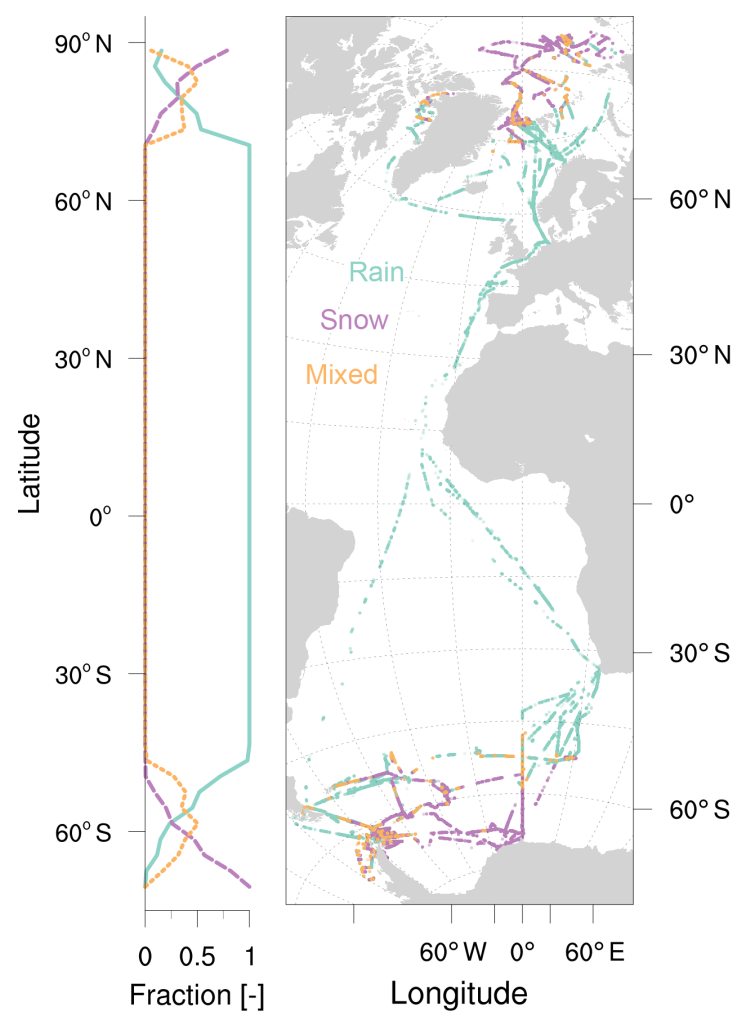

Figure 2. Map illustrates ship tracks from RV Polarstern ALL data (11 June 2010-8 October 2014), whereby dots denote minutes of occurring precipitation classified by the manual PP distinction (cyan: rain; orange: mixed phase; purple: snow). Harbor times and minutes without precipitation are not shown. Left side denotes the fraction of each PP averaged per latitude.

cluding periods of maintenance in harbors and instrument outages. Snow or mixed-phase precipitation occurred almost exclusively poleward of $45^{\circ} \mathrm{S}$ and $70^{\circ} \mathrm{N}$, which largely depends on seasonality. During boreal warm season, RV Polarstern sailed over the northern hemispheric Atlantic Ocean and in the entire Arctic area, whereas during austral warm- 
Table 2. OceanRAIN data sets from RV Polarstern divided into sub-data sets that are used in the analysis. While RSM (rain, snow, mixed phase) and ALL (all data) include the mixed phase, RS (rain, snow) sub-data exclude mixed-phase precipitation. RSM and RS include only those minutes with at least 20 particles of precipitation falling at mid- or high latitudes at air temperatures around the freezing point (see Sect. 2.3). The no-rain fraction (rain fraction subtracted from 1) yields the fraction frozen precipitation meaning snow cases for RS and snow and mixed phase for RSM and ALL.

\begin{tabular}{llrr}
\hline Name & Description & Size (min) & No rain (frac) \\
\hline ALL & Complete data sample & 268340 & 0.57 \\
RSM & $\begin{array}{l}\text { Data sub-sample } \\
\text { (incl. mixed phase) }\end{array}$ & 165632 & 0.61 \\
RS & $\begin{array}{l}\text { Data sub-sample } \\
\text { (excl. mixed phase) }\end{array}$ & 149635 & 0.57 \\
\hline
\end{tabular}

season RV Polarstern cruised on the southern hemispheric Atlantic Ocean and at the Antarctic. As an exception, RV Polarstern spent the whole year 2013 including austral cold season in the Southern Hemisphere, which explains the multitude of mixed-phase and snow precipitation cases between 45 and $75^{\circ} \mathrm{S}$ that are not sampled at corresponding northern hemispheric latitudes. For the sake of polar research, RV Polarstern spends most research time in the polar regions, which results in a high time fraction of snow or mixed-phase precipitation of 0.57 (Table 2). Accordingly, precipitation occurred most frequently at temperatures around $0{ }^{\circ} \mathrm{C}$ and at high relative humidity (Fig. 3). The high time fraction of snow or mixed-phase precipitation around $0^{\circ} \mathrm{C}$ makes $\mathrm{RV}$ Polarstern an extremely valuable data set for oceanic PP distinction analysis.

The whole RV Polarstern data set, denoted ALL (for all data), consists of about $268000 \mathrm{~min}$ of precipitation. The ship's positions cover large areas of distinctly high or low temperatures where the PP assignment is trivial and does not help in developing the PP algorithm. Therefore, we reduce the complete RV Polarstern data set ALL to minutes of highest PP uncertainty (Table 2). Air temperatures below $-6^{\circ} \mathrm{C}$ and above $8^{\circ} \mathrm{C}$ are excluded as well as ship locations between $45^{\circ} \mathrm{S}$ and $70^{\circ} \mathrm{N}$ latitude wherein virtually no solid or mixed-phase precipitation was observed within the 4-year period (Fig. 2). We exclude minutes with a total particle number of less than 20 particles because they cannot guarantee a meaningful PP distinction. These limitations leave a subset of data denoted RSM (for rain, snow, mixed phase) with 165632 min of rain, snow, or mixed-phase precipitation. By that, the no-rain time fraction including snow or mixed-phase precipitation increases from 0.57 (ALL) to 0.61 (RSM). If we further exclude mixed-phase precipitation the gained subsample, denoted RS (for rain, snow), reduces to $149635 \mathrm{~min}$ while the no-rain fraction decreases to 0.57 (Table 2).

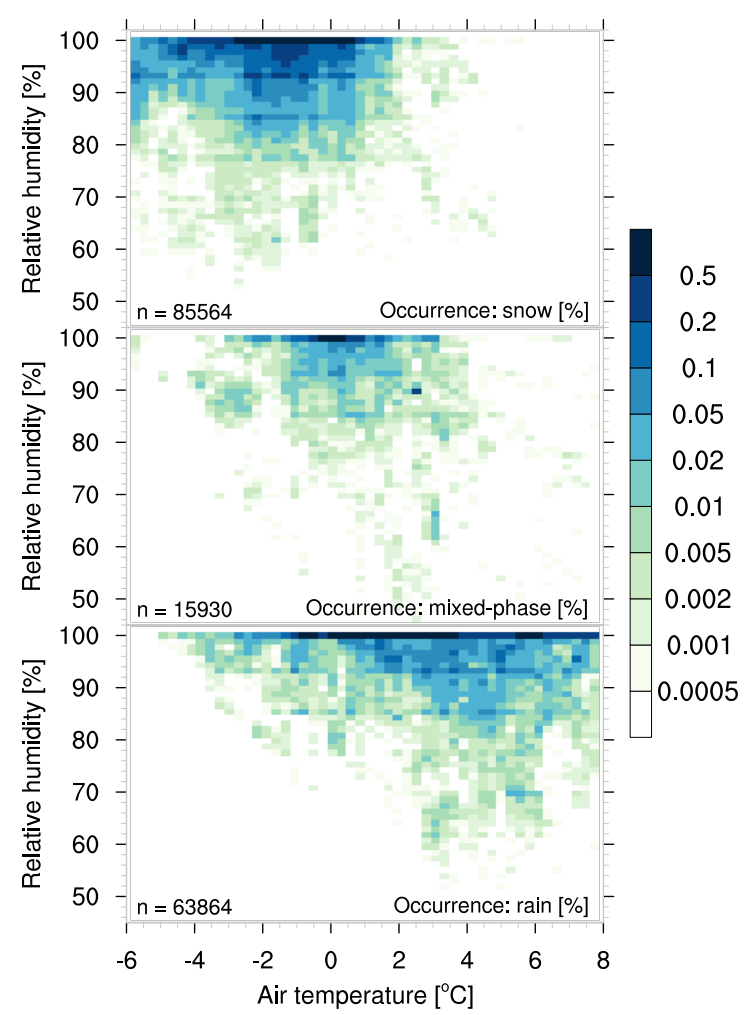

Figure 3. Two-dimensional histogram shows relative occurrence (\%) for each PP (top: snow; middle: mixed phase; bottom: rain) after manual PP distinction from OceanRAIN RSM data set of RV Polarstern. $n$ denotes the number of minutes used per PP (165 632 in total).

Atmospheric variables measured onboard RV Polarstern include temperature-related $\left(T, T_{\mathrm{d}}, T_{2 \mathrm{~h}}\right)$ and humidityrelated variables $\left(\mathrm{rH}, T_{\mathrm{d}}\right)$, air pressure $(P)$, and, from the ODM470, wind speed (not used for analysis) and particle diameter $(D)$. Instead of $D$, we use the 99th percentile of $D$, $D_{99}$, which is a measure for the maximum particle diameter measured within 1 min but excluding erroneously large particles possibly caused by particle coincidences, drip-off drops, or other artifacts. Table 3 lists all relevant variables from RV Polarstern and the ODM470. Note that all variables are measured distinctly higher than $2 \mathrm{~m}$ above the surface at about $43 \mathrm{~m}$ in order to reduce interfering sea spray and splashing wave water.

\section{The automatic PP distinction}

\subsection{One PP distribution to predict two PPs (2P1D)}

This study aims at predicting the PP automatically by using available in situ atmospheric predictor variables (Table 3). While we first focus on predicting two PPs using one PP distribution (Sect. 3.1; 2P1D), we later apply one PP distribution to predict three PPs (incl. mixed phase; Sect. 3.2; 3P1D). 
Table 3. List of available meteorological predictor variables in OceanRAIN used in the logistic regression model to predict the PP.

\begin{tabular}{llll}
\hline Variable & Description & Unit & Source \\
\hline$T$ & Air temperature & ${ }^{\circ} \mathrm{C}$ & Polarstern \\
$T_{\mathrm{d}}$ & Dew point temperature & ${ }^{\circ} \mathrm{C}$ & Polarstern \\
$T_{2 \mathrm{~h}}$ & Air temperature 2 h prior to observation & ${ }^{\circ} \mathrm{C}$ & Polarstern \\
$\mathrm{rH}$ & Relative humidity & $\%$ & Polarstern \\
$P$ & Sea-level air pressure & $\mathrm{hPa}$ & Polarstern \\
$\mathrm{RR}$ & Precipitation rate assuming rain & $\mathrm{mm} \mathrm{h}{ }^{-1}$ & ODM470 \\
$D_{99}$ & 99th percentile of particle diameter & $\mathrm{mm}$ & ODM470 \\
\hline
\end{tabular}

Section 3.3 presents a novel approach that predicts three PPs applying two PP distributions (3P2D).

For the PP prediction we adopt a statistical model using logistic regression to relate the available observed atmospheric variables (predictor variables) to the PP as suggested by Koistinen and Saltikoff (1998), henceforth KS98. The predictor variables are fitted against binary dependent variables to calculate the PP probability $p(\mathrm{PP})$. Taken from the manual PP distinction data (Sect. 2.2), the binary dependent variables attain a rain probability $p$ (rain) [frac] of either 0 (snow) or 1 (rain). Once fitted, $p$ (rain) can attain any value between 0 and 1 depending on the predictor variables. $p$ (rain) is calculated by

$p($ rain $)=\frac{1}{1+e^{\alpha+\beta \cdot V_{1}+\gamma \cdot V_{2}+\ldots+\omega \cdot V_{n}}}$,

whereby $V_{i}$ represents the atmospheric predictor variables. $\alpha, \beta, \gamma, \ldots, \omega$ denote the regression coefficients that are determined by minimizing the sum of squared errors (nearestneighbor method) with respect to the PPs from the manual PP distinction. Generally, we use the term PP probability, $p(\mathrm{PP})$, representing both rain ( $p($ rain) $)$ and snow probability $(p$ (snow)) if not stated differently. The snow probability is calculated as $1-p$ (rain), excluding mixed phase for now in this simple model.

We calibrate various combinations of atmospheric predictor variables $V_{i}$ (Table 3) for RS sub-data (Table 2) to find the combination that predicts best the PP. KS98 state that the combination of air temperature $T$ and relative humidity $\mathrm{rH}$, called $T \_\mathrm{rH}$, is suited best to predict the PP. For $T \_\mathrm{rH}$, Eq. (7) changes to

$p($ rain $)=\frac{1}{1+e^{(\alpha+\beta \cdot T+\gamma \cdot \mathrm{rH})}}$,

where the number of regression coefficients reduces to three. In lack of alternative reference data, we evaluate the calculated regression coefficients of RS sub-data using the same manually determined PPs as used for the model calibration. Nevertheless, we investigated the robustness of the regression coefficients using 100 realizations of only $50 \%$ randomly chosen minutes of precipitation from the RS data set. The standard deviation of the 100 realizations rarely exceeds
$10 \%$ of the individual regression coefficients from the whole RS data set, which confirms the robustness of the calculated regression coefficients. If in the manual PP reference data set a minute of precipitation is assigned rain, the statistical model by definition "agrees" for $p($ rain $) \geq 0.5$ while it "disagrees" for $p$ (rain) $<0.5$. For the rain/snow distinction four possible combinations exist - rain agreement, snow agreement, rain disagreement, and snow disagreement. For instance, rain disagreement means that the statistical model predicts rain that disagrees with the manual PP reference data indicating snow. Combined in a contingency table we choose four scores to evaluate how well the atmospheric predictor variable combinations serve to predict the PP in this statistical model.

First, the accuracy serves to evaluate the overall correctness of the predictor variable combinations with respect to the manual PP reference data set. The accuracy represents the sum of cases in which model and manually determined PP reference data of RS sub-data agree divided by the total number of minutes in RS sub-data. Ideally, the accuracy is close to 1 .

Second, we consider the bias score defined as the ratio between the sum of disagreeing rain predictions and agreeing rain predictions to the sum of disagreeing snow predictions and agreeing rain predictions, all with respect to the manually determined PP reference data. Accordingly, a bias score of $b<1$ represents an overprediction of snow by the model, whereas $b>1$ represents an overprediction of rain by the model. However, the bias should be interpreted with caution because the manual reference data set might be biased itself. Thus, the bias rather carries the information in which direction the predicted PP deviates from the manual reference data.

Third, we determine the percentage of cases misclassified (PM). Misclassified means that predicted highprobability cases $(p>0.95)$ disagree with the manual PP reference data. For PM, the number of these misclassified cases is divided by the number of all RS cases. Ideally, PM is close to 0 .

Fourth, the percentage of uncertain cases (PU) estimates how well the PPs are separated by the predictor variables used. PU represents the number of cases with $0.05<p<$ 
0.95 divided by all RS cases. Accordingly, PU measures the fraction of cases that the model is unable to predict at a high level of certainty. The definitions of PM and PU follow the evaluation method in Froidurot et al. (2014).

The four performance scores are calculated for both 100 realizations of $50 \%$ randomly chosen minutes of precipitation (black boxes and whiskers in Fig. 4) and for all minutes of RS sub-data (red stars). The percentiles (5th, 25th, 50th, 75th, and 95th) illustrate how strongly the RS data set scatters and whether differences among predictor variable combinations are significant $(p=0.95, n=100)$.

The PPs calculated with the logistic regression model reach an accuracy of more than $88 \%$ for combinations of atmospheric predictor variables that all include the air temperature $T$ (Fig. 4). $T$ carries the most straightforward PP information in most cases. Combining $T$ with up to two other relevant predictor variables (connected by underscores) aids to assess their value in determining the PP. Table 4 displays the most important fitted regression coefficients for different combinations of predictor variables using the OceanRAIN sub-sample RS (2P1D) and the sub-sample RSM (3P1D and 3P2D).

Combining $T$ with the air temperature $2 \mathrm{~h}$ prior to observation $\left(T_{2 \mathrm{~h}}\right)$ does not increase the accuracy of $T$ (both $88.5 \%$ ). Other time intervals led to similarly small performance changes being in agreement with Froidurot et al. (2014). Accordingly, weather fronts associated with $T$ drops do not seem to have an imprint on $T_{-} T_{2 \mathrm{~h}}$ or they are currently underrepresented in the OceanRAIN data set. The air pressure $P$ may have an impact on the $\mathrm{PP}$ at higher elevations due to lower air density (Dai, 2008). This, however, cannot explain the better accuracy of $89.2 \%$ for $T \_P$ compared to $T$. Over the ocean, the additional skill in the predictor $P$ might be caused by certain weather situations that favor either rain or snow, and are sufficiently sampled in the OceanRAIN data set. The relative humidity $\mathrm{rH}$ and the dew point $T_{\mathrm{d}}$ (not shown) reach about the same accuracy of $89.4 \%$. The addition of $P$ and $\mathrm{rH}$ to $T$ leads to a statistically significant ( $p=0.95, n=100)$ but only slight increase in accuracy compared to $T$ alone.

With the 99th percentile of the particle diameter $D_{99}$ and the calculated theoretical rain rate RR (Eqs. 3 and 4), physical properties of precipitation particles directly enter the PP distinction. This direct physical relation explains the notably higher accuracy of at least $90 \%$ by $T \_$RR, $T_{-} D_{99}$, and other combinations containing RR and $D_{99}$. The similarly high performance of these three predictor combinations is driven by the particle diameter that mainly influences RR. Combinations of $T$, a humidity-related variable such as $\mathrm{rH}$, and a diameter-related variable such as $D_{99}$ reach the highest accuracy of more than $91 \%$. Combinations of four or five of the available atmospheric predictor variables such as $T \_$rH_RR_D $D 9$ brought no noticeable further increase in accuracy (not shown). From the considered predictors, a com-

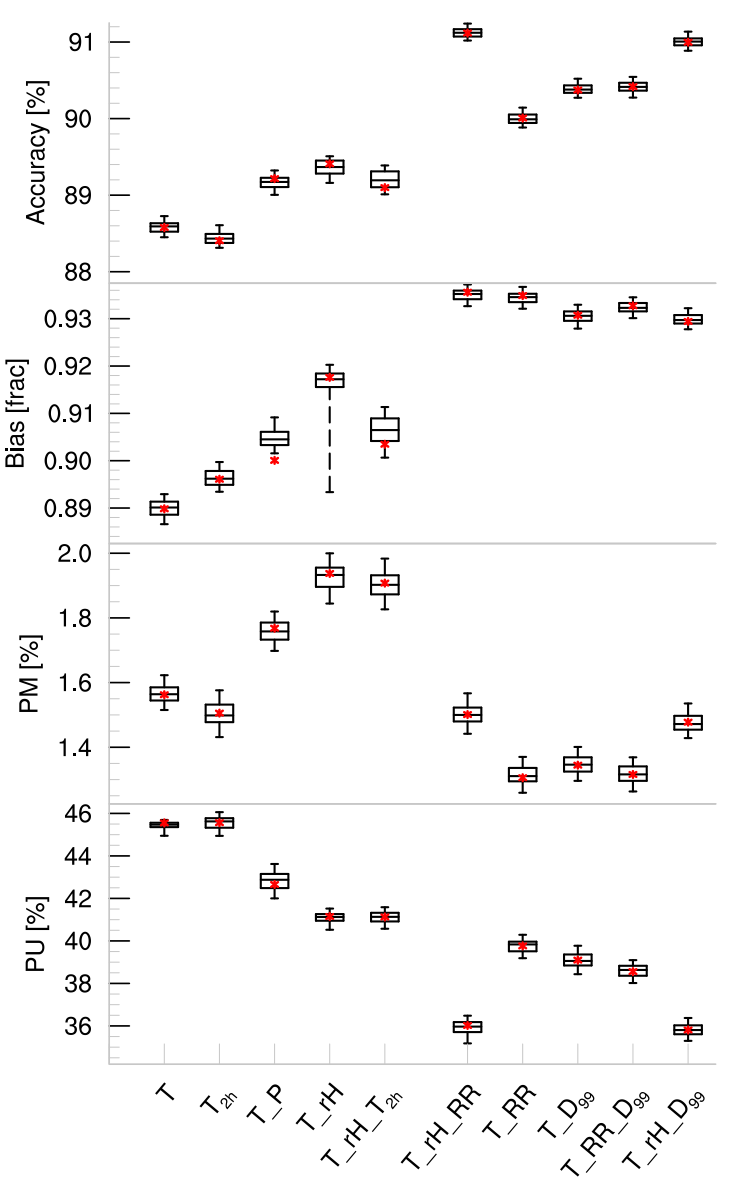

Figure 4. Box-and-whisker plot displays interquartile spread (black box: 25th, 50th, and 75th percentile) and lower (whisker: 5th percentile) as well as upper ( 95 th percentile) extremes, calculated from 100 realizations of each $50 \%$ randomly chosen minutes of precipitation from RS sub-data. Red stars denote the values for $100 \%$ of RS sub-data. Accuracy (\%), bias score (frac), percentage misclassified (PM: fraction of disagreeing cases with high certainty of $p>0.95$ in \%), and percentage unclassified (PU: fraction of uncertain cases of $0.05<p<0.95$ in $\%$ ) serve as performance scores using the calculated coefficients in Table 4 against the manually determined PP reference data. Labels indicate variable combinations, whereby all combinations include $T$.

bination of three out of the available predictor variables suits best to accurately distinguish between rain and snow.

The bias provides the ratio of rain cases predicted by the statistical model and observed rain cases from the manual PP reference data. All predictor variable combinations range between 0.89 and 0.94 , which implies an underprediction of rain and hence an overprediction of snow. Combinations that contain RR and $D_{99}$ reach the smallest overprediction of snow, whereas $T$ holds the strongest snow bias. The lower snow bias combined with the higher accuracy of predictor variables carrying particle diameter information highlights the need to include physically related variables in a statistical model to predict the PP. 
Table 4. List of regression coefficients calculated with Eq. (7) by minimizing the sum of squared errors with respect to the manual PP reference data for two PPs using one PP distribution (2P1D; Sect. 3.1), three PPs using one PP distribution (3P1D; Sect. 3.2), and three PPs using two PP distributions (3P2D; Sect. 3.3). For 3P2D, the asterisk denotes the rain distribution that was derived setting the mixed phase to snow. KS98 denotes the coefficients recommended by Koistinen and Saltikoff (1998) derived over Finland.

\begin{tabular}{|c|c|c|c|c|c|}
\hline \multirow[t]{2}{*}{ Method } & \multirow{2}{*}{$\begin{array}{l}\text { Variables used } \\
\left(V_{1} \_V_{2} \_V_{3}\right)\end{array}$} & \multicolumn{4}{|c|}{ Regression coefficients } \\
\hline & & $\alpha$ & $\beta$ & $\gamma$ & $\delta$ \\
\hline KS98 & $T$ _rH & -22 & 2.7 & 0.2 & - \\
\hline \multirow{3}{*}{ 2P1D } & $T \_r H$ & -13.39 & 1.818 & 0.127 & - \\
\hline & $T \_$rH_D $D 99$ & -10.83 & 1.780 & 0.118 & -1.062 \\
\hline & $T \_$rH_RR & -13.55 & 1.738 & 0.135 & -0.325 \\
\hline \multirow[t]{3}{*}{ 3P1D } & $T \_\mathrm{rH}$ & -9.766 & 1.382 & 0.092 & - \\
\hline & $T \_$rH_D $D_{99}$ & -8.364 & 1.364 & 0.090 & -0.732 \\
\hline & $T \_\mathrm{rH} \_\mathrm{RR}$ & -10.01 & 1.331 & 0.099 & -0.204 \\
\hline \multirow[t]{6}{*}{$3 \mathrm{P} 2 \mathrm{D}$} & $T \_\mathrm{rH}$ & -5.687 & 1.429 & 0.055 & - \\
\hline & $T \_\mathrm{rH}^{*}$ & -15.40 & 1.482 & 0.144 & - \\
\hline & $T \_$rH_D $D_{99}$ & -4.794 & 1.467 & 0.056 & -0.556 \\
\hline & $T \_r H_{-} D_{99} *$ & -13.94 & 1.431 & 0.145 & -0.959 \\
\hline & $T \_$rH_RR & -5.888 & 1.412 & 0.060 & -0.059 \\
\hline & $T \_\mathrm{rH} \_\mathrm{RR} *$ & -13.95 & 1.382 & 0.136 & -0.316 \\
\hline
\end{tabular}

Besides being accurate and unbiased, a small PP transition region of low PP certainty (low PU) combined with a low fraction of highly certain but misclassified PP cases (low PM) characterize a useful predictor variable combination. The PU decreases with increasing accuracy. Consequently, predictor variable combinations including $\mathrm{rH}$ and either $D_{99}$ or RR reach the lowest PU of about $36 \%$. This low PU and thus fairly narrow PP distribution causes a slight increase in PM for $T \_$rH_RR and $T \_$rH_ $D_{99}(1.5 \%)$ compared to $T \_D_{99}$, $T \_\mathrm{RR}$, and $T \_\mathrm{RR} \_D_{99}(1.3 \%)$. However, the positive effect of using RR or $D_{99}$ outweighs the slightly negative influence of $\mathrm{rH}$ on PM. Consequently, the physical related predictor variables confirm their good performance in predicting the PP.

The $T \_$rH coefficients that were calculated for Finland in KS98 and confirmed in Froidurot et al. (2014) over Switzerland reach an accuracy of $88.6 \%$, which is slightly lower than those coefficients optimized for OceanRAIN (89.4\%). A two-tailed $t$ test confirms the difference to be statistically significant ( $p=0.99, n=100$ ). The OceanRAIN-adapted coefficients exhibit a shallower rain/snow transition that results in a $0.8^{\circ} \mathrm{C}$ lower temperature at $p($ rain $)=0.1$ while both distributions equal at $p$ (rain) $=0.9$ (Fig. 5). Compared to OceanRAIN, the steeper rain/snow transition against $T$ fitted in KS98 holds a much lower PU of $24 \%$ but to the expense of a much higher PM of $4 \%$ and a snow bias of 0.8 . Consequently, the coefficients from KS98 better predict most uncertain cases with $T \_$rH but miss more extreme cases such as freezing rain. For the OceanRAIN data set, the PP prediction using the RS-fitted coefficients better reflects the Ocean-

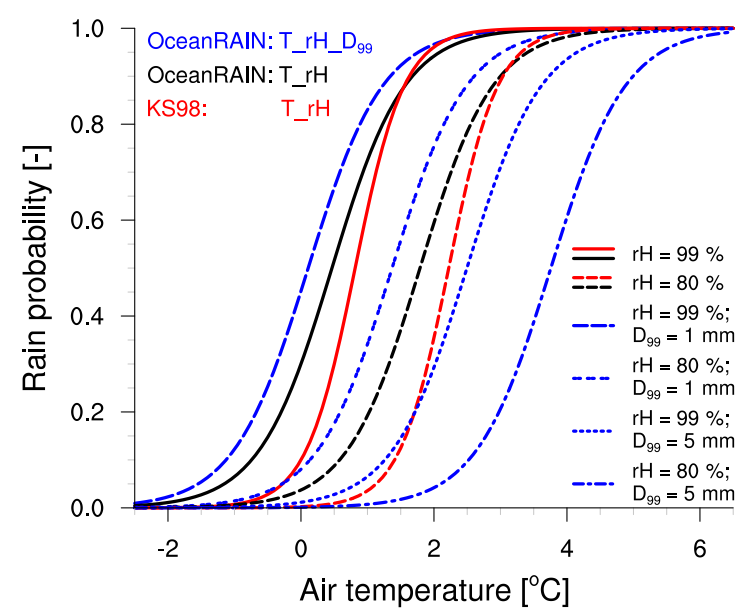

Figure 5. Rain probability using regression coefficients from Table 4 for OceanRAIN RS sub-data (2P1D) with the predictor variables $T \_$rH (black), $T \_$rH_ $D_{99}$ (blue) both fitted against OceanRAIN, compared to KS98-recommended coefficients for $T \_r H$ (red). Dashed lines (black, red) indicate a PP distribution where $\mathrm{rH}$ is set to $80 \%$ while for solid lines it is set to $99 \%$. For $T \_$rH_ $D_{99}$ (blue lines), $D_{99}$ is set to either 1 or $5 \mathrm{~mm}$ in addition to $\mathrm{rH}$.

RAIN PP distribution compared to the KS98-fitted coefficients as indicated by the accuracy.

For $T$ _rH_ $D_{99}$, the rain/snow transition shifts with $T$ depending on $D_{99}$. While $D_{99}=1 \mathrm{~mm}$ shifts the rain/snow transition to even lower temperatures by about $0.5^{\circ} \mathrm{C}, D_{99}=$ $5 \mathrm{~mm}$ shifts it towards higher temperatures by about $2{ }^{\circ} \mathrm{C}$, both compared to $T$ _rH derived from OceanRAIN RS subdata. The shallower rain/snow transition of the PP distribution fitted for OceanRAIN compared to that over Finland is likely caused by more freezing rain cases sampled in OceanRAIN, which the KS98-fitted coefficients for $T \_$rH cannot predict.

\subsection{One PP distribution to predict three PPs (3P1D)}

In a second step, we include mixed-phase precipitation into the algorithm because mixed-phase precipitation marks the transition from frozen to liquid particles and thus carries the highest uncertainty. We calculate the regression coefficients using the RSM sub-data including $165632 \mathrm{~min}$ of precipitation measured onboard RV Polarstern. The threephase distinction 3P1D fits $p$ (rain) against three PPs from the same manually determined PP reference data set as before. However, the calculated transition phase between snow with $p($ rain $)=0$ and rain with $p($ rain $)=1$ is interpreted as mixed phase, defined in the range of $0.3 \leq p$ (rain) $\leq 0.7$ after KS98. The approximated coefficients for predictor variable combinations $V_{i}$ differ considerably from those calculated for the two-phase method 2P1D (see Table 4 in Sect. 3.1).

We evaluate the calculated PP probability against PPs from the manual PP reference data using RSM sub-data. Again, 
accuracy, bias, and PM serve as a measure of quality, while $\mathrm{PU}$ is no longer suitable for evaluation because the transition region of highest uncertainty between snow and rain represents mixed-phase precipitation. Overall, this three-phase method 3P1D yields an accuracy between 74 and $78 \%$, which corresponds to an absolute decrease of about $14 \%$ compared to 2P1D (Fig. 6). To that large decrease in accuracy two reasons mainly contribute: (1) the manual PP reference data, acting as reference data, holds large uncertainties in the mixed phase, as well. The $w w$ code represents snapshots of 3-hourly observations. Therefore, they hardly satisfy the need for minute-based observations because the mixedphase rain/snow fraction can vary dramatically, both temporally and spatially. (2) KS98 assume the mixed-phase precipitation to occur in the transition region between rain and snow, which is true in most cases. However, several cases exist in which mixed-phase precipitation occurs at distinctly high or low air temperature (Fig. 3) and thus 3P1D misclassifies these cases.

Relative to each other, the individual variable combinations perform similar compared to 2P1D. $T, T T_{2} \mathrm{~h}$, and $T \_P$ have the lowest accuracy of below $75 \%$ (Fig. 6) and a bias below 0.92 . The addition of $\mathrm{rH}$ significantly increases the accuracy by about $1 \%$, whereas $T \_r{ }_{-} T_{2} \mathrm{~h}$, $T \_$rH, and $T \_T_{\mathrm{d} \_} T_{2 \mathrm{~h}}$ (not shown) do not differ much from each other. The predictor variable combinations that include the diameter-related predictors RR and $D_{99}$ lead to the highest accuracy of 76 up to $78 \%$. The highest accuracy of $78 \%$ reached by $T \_$rH_ $D_{99}$ represents a statistically significant performance increase to the remaining variable combinations in 3P1D, which contrasts to 2P1D where $T$ rrH_RR does not perform significantly better than $T \_$rH_ $D_{99}$.

For the bias, predictor combinations including RR and/or $D_{99}$ reach the least pronounced snow bias of about 0.93, whereas the remaining predictor combinations feature significantly lower biases, mostly below 0.92 . In that respect, the bias of 3P1D resembles that of 2P1D (see Fig. 4 in Sect. 3.1) both in terms of magnitude and in the individual performance of the predictor variable combinations.

While the ranking of predictor variable combinations with respect to accuracy and bias looks very similar compared to 2P1D, PM tends to form three clusters. The first cluster comprises predictor variables without particle diameter information, holding the lowest PM of 2.2 to $2.4 \%$. The second cluster includes RR but not $D_{99}$, holding the highest PM (3.4\%). In the third cluster each predictor variable combination includes $D_{99}$ but performs better than the second cluster with PM of about $2.8 \%$. $T \_$rH_ $D_{99}$ in the third cluster offers the best compromise in maximizing the accuracy while minimizing the fraction of misclassified cases.

In contrast to 2P1D, for 3P1D PM tends to scale with accuracy for many predictor variable combinations. While $T \_$rH_D $D_{99}$ exhibits an about $0.5 \%$ larger PM than $T$, the PM of $T \_$rH_RR and $T \_$RR are even $1.1 \%$ larger. A high PM indicates a clear disagreement between calculated PP

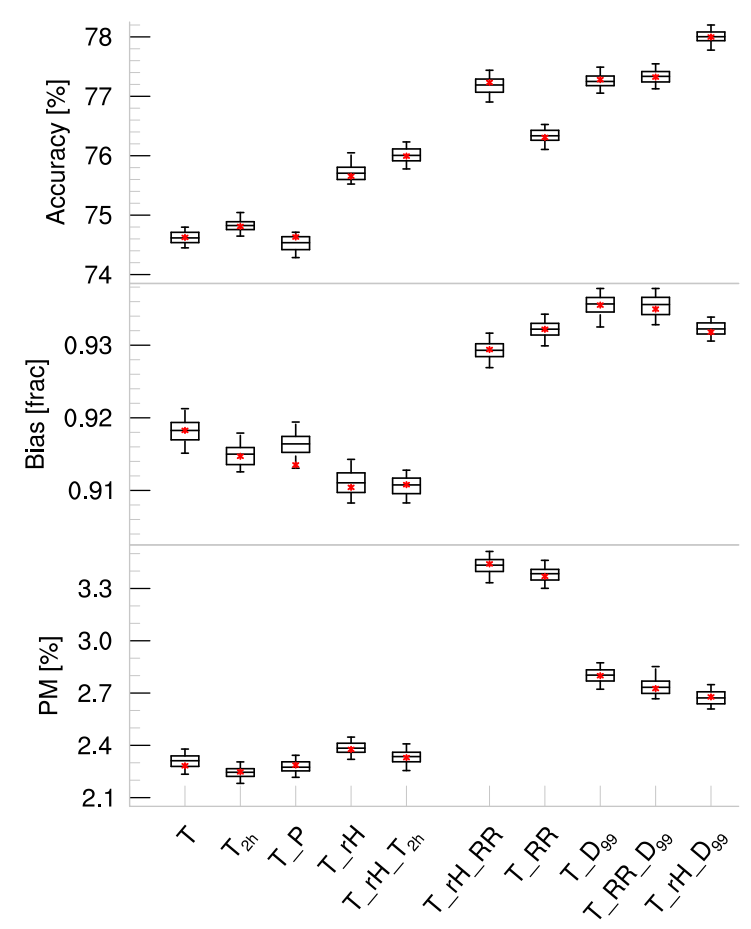

Figure 6. Performance of fit is shown for different combinations of atmospheric variables as in Fig. 4 for RSM sub-data. All variable combinations again include $T$.

and manually estimated PP. Note, however, that not in all of these clearly disagreeing cases the manual PP reference data necessarily contains the correct PP. Physically related predictor variables such as $D_{99}$ can assist to unveil cases falsely classified by the manual PP estimation. For example, $D_{99}$ is able to identify snow or mixed-phase cases, falsely classified as rain in the manual reference data. Except for the tropics, rain drops rarely exceed drop diameters of $5 \mathrm{~mm}$ (Bentley, 1904; Villermaux and Bossa, 2009). Larger drops mainly break up or collide with neighboring drops. $D_{99}$ excludes coincidences of drops as well as artificial drops dripping off the instrument housing by discarding the uppermost percentile of measured drop diameters per minute. Therefore, particles classified as rain drops with $D_{99}>5 \mathrm{~mm}$ very likely represent frozen particles, which means that they were falsely classified as rain (Fig. 7). Below $4{ }^{\circ} \mathrm{C}, 163$ rain cases in RSM subdata (about $0.25 \%$ ) are likely falsely classified. This could explain about half of the $0.5 \%$ PM difference of $T \_r H_{-} D_{99}$ to $T$ in Fig. 6).

The $T$ rH coefficients calculated in KS98 reach an accuracy of $78.6 \%$, but PM amounts to $7.2 \%$ misclassified cases (not shown), which is more than a factor of 2.5 higher than the PM of $T \_$rH_ $D_{99}$. The shift towards higher air temperatures and the steeper rain/snow transition in the PP distribution using the coefficients recommended in KS98 (see Fig. 5) explain the large amount of misclassified cases. However, as stated before, the coefficients in KS98 derived over Finland 
cannot represent the temperature distribution of PPs in the OceanRAIN data set.

\subsection{Two PP distributions to predict three PPs (3P2D)}

The relatively low accuracy reached with the three-phase method after KS98 using one PP distribution (3P1D) motivates a novel investigation of how to further improve the PP prediction for three PPs. Instead of applying one PP distribution to determine rain, mixed-phase, and snow precipitation, we suggest to approximate two separate PP distributions for rain and snow (3P2D). These two individual PP distributions are derived analogous to the method for one PP distribution by assigning the mixed-phase PP differently - first set it to rain to calculate the snow PP distribution, then set it to snow to calculate the rain PP distribution. Subtracting the sum of both individually calculated PP distributions from 1 yields the PP distribution for mixed phase. In contrast to 3P1D, the separately calculated coefficients for rain and snow (Table 4) lead to individual distributions only connected via the mixed phase.

Analogous to 2P1D (Sect. 3.1), the accuracy represents the percentage of cases with $p(\mathrm{PP})>0.5$ that agree in their respective $\mathrm{PP}$ with the manual $\mathrm{PP}$ reference data. The bias represents the ratio between the sum of predicted rain cases and the sum of rain cases in the manual PP reference data. Please note that the bias definition remains unchanged for 3P2D that includes mixed phase compared to 2P1D. However, the additional PP distribution slightly modifies the calculation of PM and PU, illustrated in Fig. 8. PM represents the percentage of all certain cases $(p(\mathrm{PP})>0.95$; hatched area in Fig. 8) in which either one of the PPs disagrees with the manual PP reference data. PU as the percentage of uncertain cases $(0.05<p(\mathrm{PP})<0.95$; shaded area) represents only those cases where all PPs are uncertain after definition. We introduce this limitation because if $p(\mathrm{PP})<0.05$ holds for at least one PP then we would not consider this PP uncertain anymore. Note that for mathematical reasons we cannot display $\mathrm{PM}_{\text {mix }}>0$ and $\mathrm{PU}>0$ in the same figure, which is why we set $\mathrm{PM}_{\text {mix }}>0$.

This 3P2D method using two individual PP distributions reaches on average a higher accuracy compared to 3P1D (Fig. 9). Whereas $T, T_{-} T_{2 \mathrm{~h}}$, and $T_{-} P$ hold less than $78 \%$ accuracy, $T \_$rH_D $D_{99}$ reaches the highest accuracy of $81.2 \%$. As for 3P1D, the improvement is mainly caused by adding the predictor $D_{99}$ that performs significantly better than when adding the predictor RR. Also the overprediction of snow by all predictor variable combinations with respect to the manually determined PP reference data stays the same in 3P2D. The physically related variables are least biased (about 0.93 ), which consistently highlights the improvement of including them in the predictor variable combination. However, for PM stronger differences among these physically related predictor variables arise. While $T \_$RR holds the highest PM (about $2.3 \%), T \_r H \_D_{99}$ reaches $1.9 \%$ PM, which is on the or-

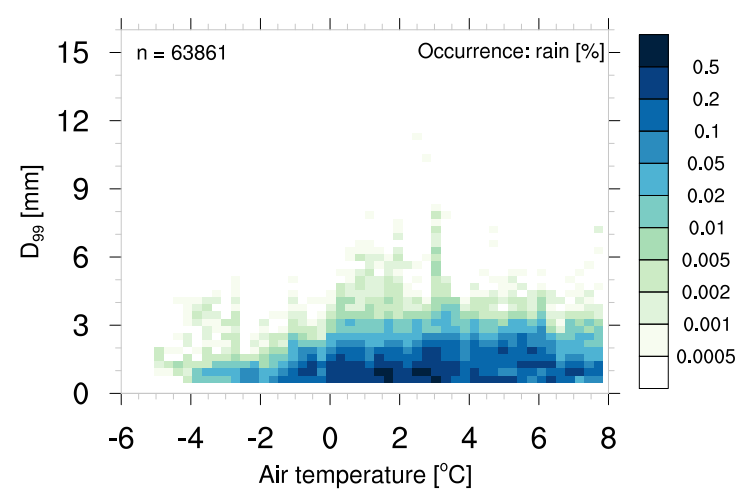

Figure 7. Two-dimensional histogram of temperature and the 99th percentile of the particle diameter for cases classified as rain by the manual PP estimation in RSM.

der of the predictor variable combinations without RR and $D_{99}(1.8 \%)$. However, the physically related predictors reach again lowest PUs of about $38 \%$ while $T$ holds a PU of $51 \%$. In combination with the other scores we recommend $T \_r H \_D 99$ followed by $T \_$RR_ $D 99$ and $T \_D 99$ to most accurately predict the PP using two independent PP distributions.

Compared to 3P1D after KS98, the PM decreases for 3P2D. This decrease in PM ranges between 0.5 and $1 \%$ and thus highlights the improved performance of using two PP distributions instead of one to predict the PP. The lower PM and higher accuracy approve that the novel method applying two independent PP distributions better represents the PP distribution in OceanRAIN RSM.

To understand the better performance of 3P2D compared to 3P1D after KS98, we consider how the PP fraction is distributed with respect to $T$ around the freezing point (rain/snow transition) in the manual PP reference data (Fig. 10). While the rain occurrence shows a relatively low skewness, the mixed-phase/snow distribution is slightly leftskewed. This higher skewness with a secondary maximum in the mixed-phase distribution at $-3{ }^{\circ} \mathrm{C}$ (minimum in snow distribution) cannot be well represented by one PP distribution. One PP distribution is limited to match all three PP distributions at the same time that can only represent an average skewness. In that respect, deriving two independent PP distributions driven by mixed-phase precipitation better reflects the PP distribution of each PP individually with respect to the manual PP reference data in OceanRAIN RSM.

The question arises whether the left-skewed distribution of snow and mixed-phase precipitation in OceanRAIN subdata RSM represents a feature of the oceanic PP distribution or if it simply reflects a currently insufficient length of the OceanRAIN time series. Though the latter seems more likely, addressing this question comprehensively, however, remains beyond the scope of this study due to the limited available OceanRAIN data sample. Future studies could clarify this 


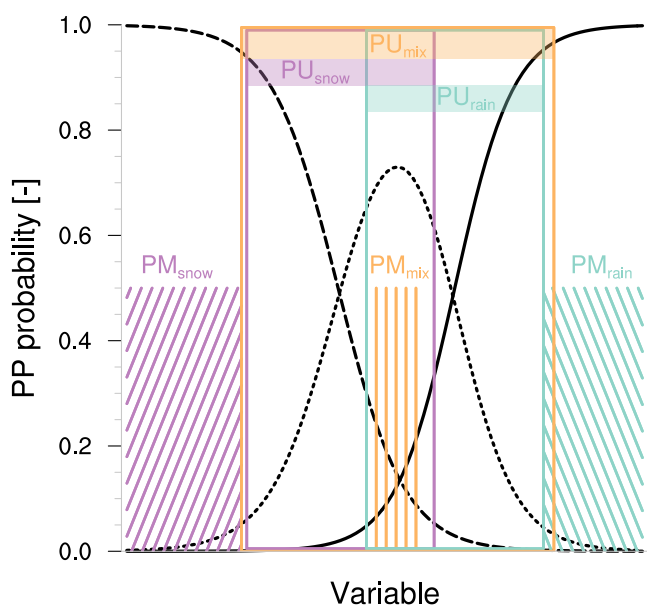

Figure 8. Graph illustrates the calculation of PU (framed) and PM (hatched) including snow (dashed/purple), mixed phase (dotted/orange), and rain (solid/cyan), analogous to Fig. 3 in Froidurot et al. (2014). PU divides the sum of cases with $0.05<p(\mathrm{PP})<0.95$ for all PPs by the sum of all RSM cases. PM divides the sum of cases with $p(\mathrm{PP})>0.95$ for one of the PPs that disagrees with the manual $\mathrm{PP}$ estimation by the sum of all RSM cases. We set $\mathrm{PM}_{\text {mix }}>0$ because otherwise we could not display it in the same PP distribution (rH kept constant) with PU $>0$.

aspect by reanalyzing the constantly growing OceanRAIN database.

Nevertheless, differences remain due to the chosen PP distinction method. By discriminating three PPs, 3P1D and 3P2D enable a smoother rain/snow transition compared to 2P1D due to included mixed-phase precipitation (Fig. 11). At lower temperatures, 2P1D approaches the snow distribution of 3P2D, while at higher temperatures it approaches the rain distribution of 3P2D. In other words, the steeper rain probability distribution for 2P1D clarifies the slightly smaller unclassified range $(0.3<p(\mathrm{PP})<0.7)$ compared to 3P2D as seen in the percentage unclassified (compare Fig. 4 and Fig. 9).

$D_{99}$ as additional variable in $T \_$rH_ $D_{99}$ tends to shift the snow and rain distributions to higher temperatures and apart of each other, which also resolves more extreme cases. This distribution shift with temperature follows a physical reason: large snow particles better withstand melting at high air temperatures than small snow particles. This physical information lacks in $T \_r H$, which notably decreases its accuracy (cf. Fig. 9).

\section{Discussion}

After finding suitable methods for both the rain/snow distinction (Sect. 3.1) as well as for the rain/snow/mixed-phase distinction (Sect. 3.3) we compare the results to those of similar studies. For the rain/snow distinction over Switzerland using $T \_$rH derived over Finland by KS98, Schmid and Mathis

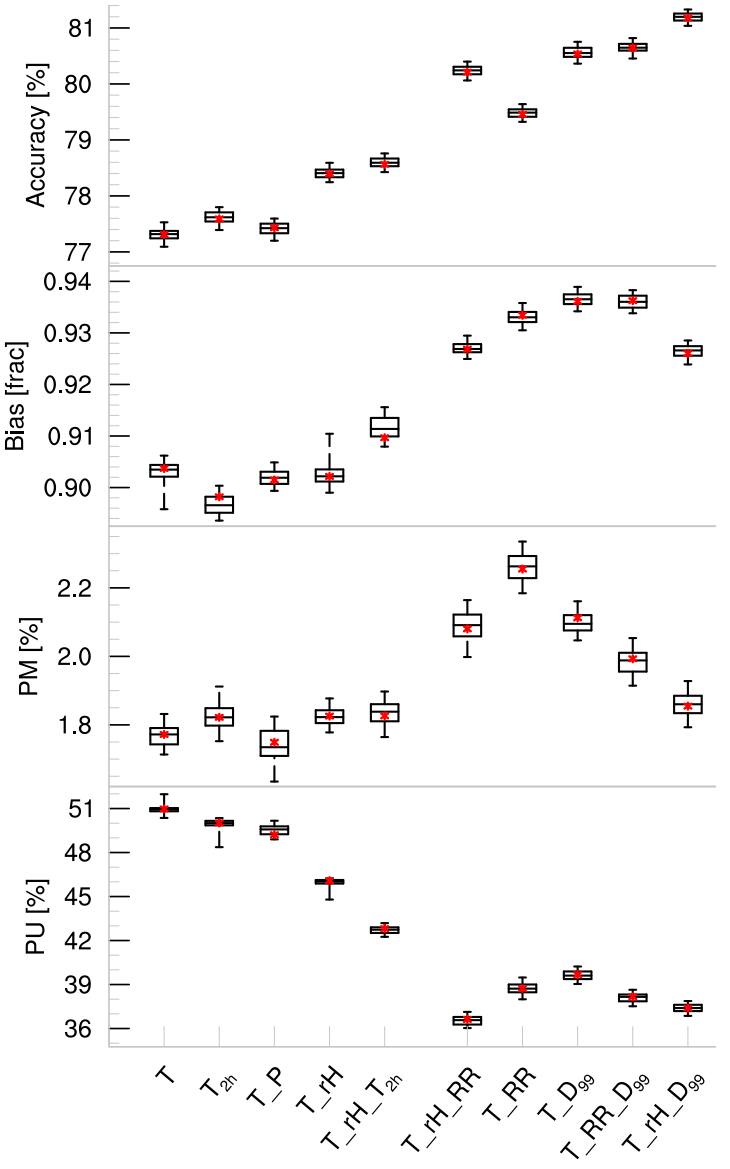

Figure 9. As Fig. 4 but for RSM including mixed phase, using two independent PP distributions (3P2D). The calculation of PM and PU differs from Fig. 4 as displayed and explained in Fig. 8.

(2004) find a higher accuracy of $92.4 \%$ compared to our calculated accuracy of $88.6 \%$ when using the same KS98 regression coefficients $\alpha=22, \beta=2.7, \gamma=0.2$. Schmid and Mathis (2004) find an overprediction of snow cases (bias 0.82 ), very similar to the OceanRAIN RS snow overprediction (bias 0.8) using the same coefficients derived by KS98. However, for fitting the regression coefficients to our data set (Table 4) we still obtain a slightly lower accuracy of $89.4 \%$ calculated for $T \_$rH and $91 \%$ for $T \_r H \_D 99$ while the lowbias decreases to 0.92 and 0.93 , respectively. These performance improvements indicate, first, different conditions for PP transition over the ocean compared to Finland of KS98 while, second, the OceanRAIN data set is in relatively close agreement with the Swiss data.

With respect to two PPs, including the mixed phase decreases the accuracy to below $78 \%$ while PM almost doubles. To elaborate on reasons for that accuracy decrease we consider a study of Gjertsen and Ødegaard (2005), who applied the same translation of $w w$ codes into PPs for $w w$ codes between 50 and 86 . Using 3P1D, they find an accuracy of $86 \%$ compared to Norwegian synoptic stations (6 months 


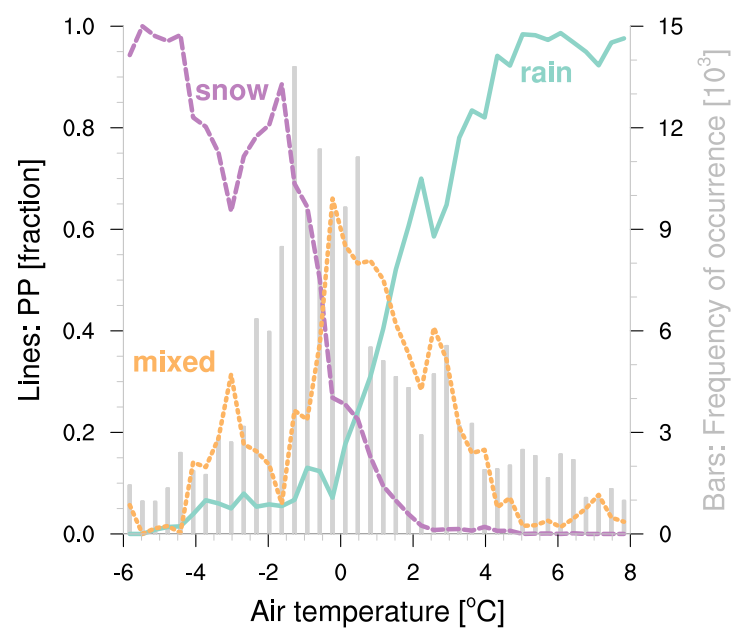

Figure 10. Lines show PP fraction for rain (solid, cyan), mixed phase (dotted, orange), and snow (purple, dashed) from OceanRAIN RSM (165632 min) determined with the manual PP estimation against temperature. Gray bars represent the temperature frequency of occurrence (in $10^{3}$ ).

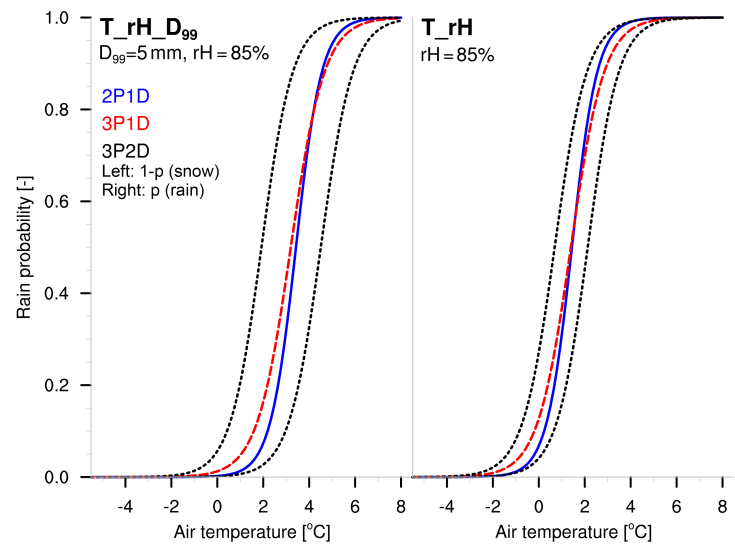

Figure 11. Air temperature versus predicted PP by the different methods: two PPs (2P1D; solid blue), three one-PP distributions (3P1D; dashed red), and three two-PP distributions (3P2D; dotted black). 3P2D consists of two curves (left: snow distribution as $1-p$ (snow); right: rain distribution as $p$ (rain)) for the calculated coefficients of $T \_r H \_D_{99}$ (left panel; $\mathrm{rH}=85 \%, D_{99}=5 \mathrm{~mm}$ ) and $T \_$rH (right panel; $\mathrm{rH}=85 \%$ ).

winter period) and $85 \%$ compared to independent climatological stations over Norway (1 month). They obtain an overprediction of snow (bias of 0.92) and problems in predicting the PP of supercooled rain during prevailing temperature inversions. In OceanRAIN we find a similar overprediction of snow (bias $T \_$rH: $0.91 ; T \_r H \_D 99: 0.93$ ) with respect to the manual PP reference data in OceanRAIN. This overprediction of snow occurs predominantly around $0{ }^{\circ} \mathrm{C}$ that is the temperature range sampled most frequently (cf. Fig. 10). Hence, OceanRAIN is likely to face the same problems underpredicting rain when supercooled raindrops fall

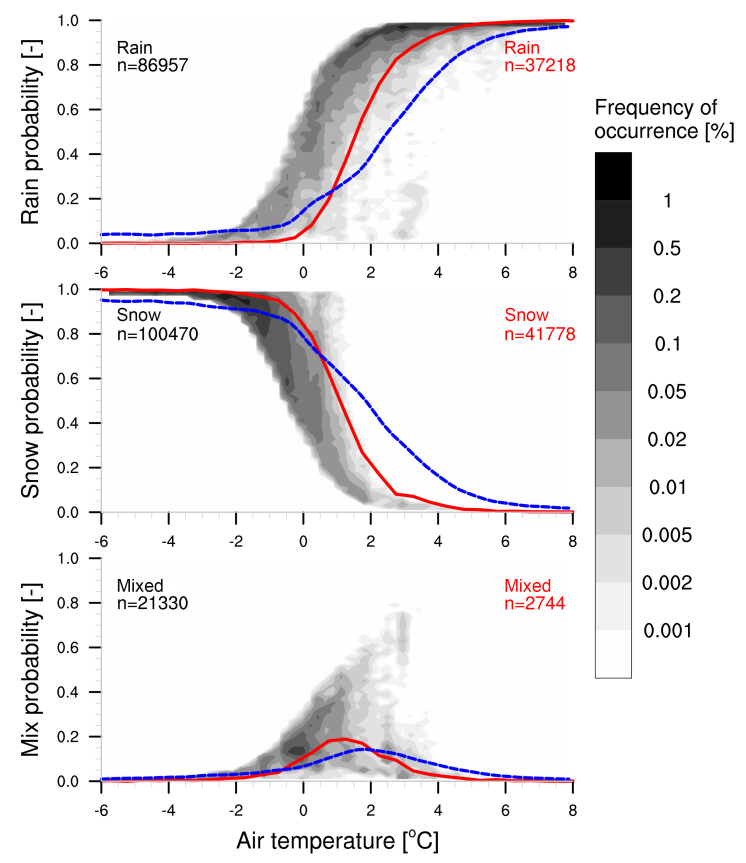

Figure 12. PP probability shown using the new 3P2D method with two individual PP distributions ( $T \_$rH_ $D 99$ ) as frequency of occurrence $(\%)$ in gray shades against air temperature according to PP reference data that separates rain, snow, and mixed phase in OceanRAIN ALL for more than 4 years of RV Polarstern data. Solid red lines represent the mean PP fraction from observations in the Swiss Alps (1991-2010) from Froidurot et al. (2014); dashed blue lines show mean PP fraction for oceanic ship data (DS464.0; 1977-2007) from Dai (2008).

under prevailing temperature inversions. Further work is required in order to clarify whether we need additional ancillary data to reduce the bias or whether the logistic regression model is unable to provide a less biased PP prediction.

Assuming that mixed-phase precipitation causes most of the accuracy decrease between 2P1D and 3P1D as well as 3P2D, we consider the individual probability of detection (POD) for rain, snow, and mixed phase. For rain, the POD is calculated by dividing the number of agreeing rain cases by the number of all observed rain cases. For the POD of 3P1D using the KS98-fitted coefficients for $T \_r H$ for rain, snow, and mixed phase we find $0.92,0.78$, and 0.21 ( $T \_r H \_D 99: 0.92,0.86$, and 0.25 ). The respective PODs from Gjertsen and Ødegaard (2005) for the same settings reveal slightly different PODs of $0.81,0.97$, and 0.25 . Whereas they obtain a notably higher POD for snow, the rain POD is lower compared to OceanRAIN. Nevertheless, mixed-phase precipitation confirms to carry the largest prediction uncertainty of all three PPs.

The variable combination $T \_$rH_ $D_{99}$ distinguishes best rain, snow, and mixed-phase precipitation in OceanRAIN data. In comparison with PP fractions allocated into temperature bins from 30 years of Swiss Alps data Froidurot et al. 
(2014), in most cases the PP transition in OceanRAIN occurs at lower temperatures (Fig. 12). However, the analysis by Froidurot et al. (2014), among other conditions, neglects all kinds of freezing rain $(w w=56,57,66,67)$ that we assign to rain. Without these "cold rain" cases, the rain/snow transition shifts towards higher temperature that may in parts explain the temperature difference in Fig. 12. Additionally, the PP probability distribution in the OceanRAIN RV Polarstern data sample is biased by the high number of temperatures around $0^{\circ} \mathrm{C}$ that occur by a factor of 3 to 4 more often than temperatures between -4 and $4{ }^{\circ} \mathrm{C}$ (cf. Fig. 10), and relative humidity close to $100 \%$. These frequently sampled conditions put their mark on the average rain/snow transition by reducing the rain/snow transition temperature compared to the Swiss Alps where $T$ and $\mathrm{rH}$ were sampled more homogeneously (Fig. 9 in Froidurot et al., 2014). Despite the high number of available minutes with precipitation in OceanRAIN, the rather short time series on climatological timescales and the spatial distribution of along-track data limit the representativeness. However, a different landocean rain/snow transition might be observable. Dai (2008) found a systematic land-ocean difference in the rain/snow transition between land and ocean in 30 years of NCEP ADP Operational Global Surface Observations (DS464.0; 1977-2007). Whereas over land, rain transitions into snow relatively quickly $\left(-2<T<4^{\circ} \mathrm{C}\right)$, over ocean the transition zone is wider $\left(-3<T<6^{\circ} \mathrm{C}\right)$. Although the rain/snow transition zone within OceanRAIN appears wider compared to regression coefficients recommended by Koistinen and Saltikoff (1998) as seen in Fig. 5, the rain/snow transition in OceanRAIN compares better to the Swiss Alps data (Froidurot et al., 2014) than to the NCEP DS464.0 ocean data (Dai, 2008) that reveal a wider transition zone. In specific, OceanRAIN relatively closely agrees with the NCEP DS464.0 ocean data for $T<0^{\circ} \mathrm{C}$, whereas larger differences of $>1{ }^{\circ} \mathrm{C}$ occur in the range of $2<T<5^{\circ} \mathrm{C}$. Two main reasons can explain the different rain/snow transitions between OceanRAIN and NCEP DS464.0 ocean data by Dai (2008). (1) $w w$ codes used in the NCEP ocean data are subject to larger uncertainty compared to OceanRAIN. In contrast to the RV Polarstern onboard weather observatory by the German Meteorological Service, many VOSs such as cargo ships in NCEP DS464.0 ocean data have inadequately trained observers that might use certain $w w$ codes preferentially, ships possibly avoid bad weather, or measurement quality may suffer from instrument biases (Petty, 1995). For these reasons, the wider rain/snow transition zone likely reflects a higher uncertainty of the NCEP DS464.0 ocean data compared to the OceanRAIN data from RV Polarstern or the Swiss Alps data. (2) RV Polarstern mainly sampled warm-season precipitation in the Atlantic Arctic and Antarctic regions with the exception of the austral cold season in 2013. In addition to that, the heterogeneous regional and seasonal sampling by RV Polarstern might have favored conditions under which inversions prevail that allow rain at fairly low temper- atures but inhibit snow under relatively high temperatures. While the sampling imbalance of RV Polarstern may indicate a restricted representativeness of PPs in OceanRAIN, the $T \_$rH_ $D 99$ predictor variable combination recommended as the new automatic PP distinction method for OceanRAIN well represents the observed PPs within OceanRAIN. The continuously growing time series of RV Polarstern among other RVs in OceanRAIN allows to recalibrate or refine the algorithm geographically for a longer time series with comprehensive statistical sampling in the future.

\section{Summary and concluding remarks}

We developed a novel automatic algorithm to distinguish the PP within OceanRAIN in situ precipitation data to introduce a statistical PP probability and to increase the data post-processing efficiency. The analysis focused on identifying the most suitable combination of available atmospheric predictor variables to determine the PP. For that purpose, we applied a simple logistic regression model suggested by Koistinen and Saltikoff (1998) that was shown to perform well over land. Previous studies mainly rely on air temperature $T$, relative humidity $\mathrm{rH}$, air pressure $P$, and others to predict the PP. We investigated several of these atmospheric predictor variable combinations to obtain a PP probability. In particular, we tested the performance of the logistic regression model after Koistinen and Saltikoff (1998) for OceanRAIN using two (excl. mixed phase) and three PPs (incl. mixed phase) against the manually estimated observationbased PP in OceanRAIN. Besides increasing the efficiency in predicting the PP with an automatic method, we developed a novel three-phase method that uses two individual and independent PP distributions to predict the PP more accurately.

The study led to the following main results.

a. In OceanRAIN, the combination of air temperature $T$, relative humidity $\mathrm{rH}$, and the 99th percentile of the particle diameter $D_{99}$ (called $T \_$rH_ $D_{99}$ ) predicts best the PP for all investigated methods.

b. Applying more than three of the chosen atmospheric predictor variables negligibly increases the accuracy in predicting the PP.

c. The two-phase method (2P1D) using the predictor variable combination $T \_$rH_ $D_{99}$ and regression coefficients fitted to OceanRAIN reaches an accuracy above $91 \%$ with a slight overestimation of snow cases for the midand high latitudes between -6 and $8^{\circ} \mathrm{C}$ in the OceanRAIN data set with respect to the manual PP reference data including shipboard present weather observations.

d. A novel three-phase method using two individual PP distributions (3P2D) for rain and snow performs better than a three-phase method that relies exclusively on one PP distribution (3P1D after Koistinen and Saltikoff, 
1998). As a reason, two individual PP distributions are capable of better representing unequally distributed or skewed PP distributions of atmospheric predictor variables as well as certain weather situations that might currently be over- or undersampled. Accordingly, this performance difference might decrease once the investigated 4-year OceanRAIN time series grows further while sampling biases vanish.

e. The OceanRAIN data using 3P2D reveal a wider rain/snow transition zone compared to data derived over Finland (Koistinen and Saltikoff, 1998). The rain/snow transition in OceanRAIN occurs at slightly lower temperatures compared to the data from Finland as well as NCEP DS464.0 global ocean ship data (Dai, 2008). The difference in the rain/snow transition zone likely originates from heterogeneous spatial and seasonal sampling in OceanRAIN that is likely to decrease with an increasing OceanRAIN time series. In contrast, a higher quality of the derived $w w$ codes in OceanRAIN compared to the average VOS suggests a higher certainty of the derived PPs. The Swiss Alps data (Froidurot et al., 2014) shows a similar rain/snow transition at slightly higher temperatures, likely caused by neglected cases of freezing rain, among others. Due to these differences we obtain the highest accuracy and lowest bias when applying regression coefficients fitted to the OceanRAIN data set instead of using recommended coefficients from the literature such as those from Koistinen and Saltikoff (1998).

f. The new PP distinction algorithm 3P2D including $D_{99}$ as essential physical information identified several cases that were erroneously classified as rain within the manual PP estimation. Large particle diameters indicate that the PP should be classified as snow or at least mixedphase precipitation instead of rain.

g. Mixed-phase precipitation carries the largest uncertainty of the three PPs and is most challenging to detect for the new algorithm with a probability of detection of up to 0.25 using the predictor variable combination $T \_$rH_ $D_{99}$ and 3P2D.

Even though the newly developed automatic PP distinction algorithm strongly depends on the currently still limited OceanRAIN data set, remarkable improvements are made. First, a PP probability is provided on a minute basis that limits the number of highly uncertain cases requiring visual inspection of atmospheric variables. The PP probability further allows error characterizing other precipitation data sets such as satellite data using OceanRAIN precipitation rates to unveil systematic errors with respect to PP. Second, the PPs of a few critical cases could be corrected that were falsely classified by the manual method. Third, we give evidence that the particle diameter of the falling precipitation particles contributes valuable information to the PP separation and by that in a physical way significantly improves the algorithm accuracy. Fourth, the new PP distinction algorithm considerably speeds up the data processing within OceanRAIN, which is an important step towards a fast-growing global surface precipitation data set for validating and evaluating other oceanic precipitation data sets.

\section{Data availability}

The OceanRAIN data set is publicly available upon request free of charge. A registration with a digital object identifier is planned. Further information are available on http: //oceanrain.org.

Acknowledgements. We would like to thank the crew of RV Polarstern including the German Meteorological Service and the Alfred Wegener Institute for their support and assistance in supervising the instrument and caring for a smooth operation and ship-board access. We further thank Nicole Albern for carefully processing the data. Helpful comments by Bjorn Stevens are well appreciated to finalize the manuscript. We also like to thank two anonymous reviewers for their valuable comments. We acknowledge the financial support of the German Research Foundation in research unit FOR1740.

The article processing charges for this open-access publication were covered by the Max Planck Society.

Edited by: M. Kulie

\section{References}

Adler, R. F., Gu, G., and Huffman, G. J.: Estimating Climatological Bias Errors for the Global Precipitation Climatology Project (GPCP), J. Appl. Meteorol. Clim., 51, 84-99, doi:10.1175/JAMC-D-11-052.1, 2012.

Atlas, D. and Ulbrich, C.: The physical basis for attenuation-rainfall relationships and the measurement of rainfall parameters by combined attenuation and radar methods, J. Rech. Atmos., 8, 275-298, 1974.

Battaglia, A., Rustemeier, E., Tokay, A., Blahak, U., and Simmer, C.: PARSIVEL Snow Observations: A Critical Assessment, J. Atmos. Ocean. Tech., 27, 333-344, doi:10.1175/2009JTECHA1332.1, 2010.

Bentley, W.: Studies of Raindrops and Raindrop Phenomena, Mon. Weather Rev., 32, 450-456, doi:10.1175/15200493(1904)32<450:SORARP>2.0.CO;2, 1904.

Bumke, K. and Seltmann, J.: Analysis of Measured Drop Size Spectra over Land and Sea, ISRN Meteorology, 2012, 1-10, doi:10.5402/2012/296575, 2012.

Clemens, M.: Machbarkeitsstudie zur räumlichen Niederschlagsanalyse aus Schiffsmessungen über der Ostsee, Berichte aus dem Institut für Meereskunde an der Christian-Albrechts-Universität Kiel, 1-50, doi:10.3289/ifm_ber_327, 2002. 
Dai, A.: Temperature and pressure dependence of the rain-snow phase transition over land and ocean, Geophys. Res. Lett., 35, L12802, doi:10.1029/2008GL033295, 2008.

Friedrich, K., Higgins, S., Masters, F. J., and Lopez, C. R.: Articulating and Stationary PARSIVEL Disdrometer Measurements in Conditions with Strong Winds and Heavy Rainfall, J. Atmos. Ocean. Tech., 30, 2063-2080, doi:10.1175/JTECH-D-1200254.1, 2013.

Froidurot, S., Zin, I., Hingray, B., and Gautheron, A.: Sensitivity of Precipitation Phase over the Swiss Alps to Different Meteorological Variables, J. Hydrometeorol., 15, 685-696, doi:10.1175/JHM-D-13-073.1, 2014.

Fuchs, T., Rapp, J., Rubel, F., and Rudolf, B.: Correction of synoptic precipitation observations due to systematic measuring errors with special regard to precipitation phases, Phys. Chem. Earth Pt. B, 26, 689-693, doi:10.1016/S1464-1909(01)00070-3, 2001.

Gjertsen, U. and Ødegaard, V.: The water phase of precipitation a comparison between observed, estimated and predicted values, Atmos. Res., 77, 218-231, doi:10.1016/j.atmosres.2004.10.030, 2005.

Goodison, B. E.: Accuracy of Canadian Snow Gage Measurements, J. Appl. Meteorol., 17, 1542-1548, doi:10.1175/15200450(1978)017<1542:AOCSGM>2.0.CO;2, 1978.

Großklaus, M.: Niederschlagsmessung auf dem Ozean von fahrenden Schiffen, PhD thesis, Institut für Meereskunde, ChristianAlbrechts-Universität Kiel, Kiel, Germany, 1996.

Hogan, A. W.: Objective Estimates of Airborne Snow Properties, J. Atmos. Ocean. Tech., 11, 432-444, doi:10.1175/15200426(1994)011<0432:OEOASP>2.0.CO;2, 1994.

Ishizaka, M., Motoyoshi, H., Nakai, S., Shiina, T., Kumakura, T., and Muramoto, K.-i.: A New Method for Identifying the Main Type of Solid Hydrometeors Contributing to Snowfall from Measured Size-Fall Speed Relationship, J. Meteorol. Soc. Jpn., 91, 747-762, doi:10.2151/jmsj.2013-602, 2013.

Kent, E. C., Ball, G., Berry, D. I., Fletcher, J., Hall, A., North, S., and Woodruff, S.: The Voluntary Observing Ship (VOS) Scheme, European Space Agency, 518-528 doi:10.5270/OceanObs09.cwp.48, 2010.

Kidd, C. and Levizzani, V.: Status of satellite precipitation retrievals, Hydrol. Earth Syst. Sci., 15, 1109-1116, doi:10.5194/hess-15-1109-2011, 2011.

Klepp, C.: The oceanic shipboard precipitation measurement network for surface validation - OceanRAIN, Atmos. Res., 163, 74-90, doi:10.1016/j.atmosres.2014.12.014, 2015.

Klepp, C., Bumke, K., Bakan, S., and Bauer, P.: Ground validation of oceanic snowfall detection in satellite climatologies during LOFZY, Tellus A, 62, 469-480, doi:10.1111/j.16000870.2010.00459.x, 2010.

Koistinen, J. and Saltikoff, E.: Eperience of customer products of accumulated snow, sleet and rain, COST75 Advanced Weather Radar Systems, 1998.

Lanza, L. G. and Vuerich, E.: The WMO Field Intercomparison of Rain Intensity Gauges, Atmos. Res., 94, 534-543, doi:10.1016/j.atmosres.2009.06.012, 2009.

Lempio, G. E., Bumke, K., and Macke, A.: Measurement of solid precipitation with an optical disdrometer, Adv. Geosci., 10, 9197, doi:10.5194/adgeo-10-91-2007, 2007.
Löffler-Mang, M. and Joss, J.: An Optical Disdrometer for Measuring Size and Velocity of Hydrometeors, J. Atmos. Ocean. Tech., 17, 130-139, doi:10.1175/15200426(2000)017<0130:AODFMS>2.0.CO;2, 2000.

Merenti-Välimäki, H.-L., Lönnqvist, J., and Laininen, P.: Present weather: comparing human observations and one type of automated sensor, Meteorol. Appl., 8, 491-496, doi:10.1017/S1350482701004108, 2001.

Michelson, D. B.: Systematic correction of precipitation gauge observations using analyzed meteorological variables, J. Hydrol., 290, 161-177, doi:10.1016/j.jhydrol.2003.10.005, 2004.

Ocean Rainfall And Ice phase precipitation measurement Network (OceanRAIN): precipitation particle spectra, precipitation rates and ancillary data from RV Polarstern over the Atlantic Ocean including polar regions, University Hamburg, CliSAP/CEN, Hamburg, Germany, available at: http://oceanrain.org, last access: 7 April 2016.

Petty, G. W.: Frequencies and Characteristics of Global Oceanic Precipitation from Shipboard Present-Weather Reports, B. Am. Meteorol. Soc., 76, 1593-1616, doi:10.1175/15200477(1995)076<1593:FACOGO>2.0.CO;2, 1995.

Schmid, W. and Mathis, A.: Validation of methods to detect winter precipitation and retrieve precipitation type, 12th SIRWEC Conference, 16-18 June 2004, Bingen, Germany, 2004.

Schneider, U., Becker, A., Finger, P., Meyer-Christoffer, A., Ziese, M., and Rudolf, B.: GPCC's new land surface precipitation climatology based on quality-controlled in situ data and its role in quantifying the global water cycle, Theor. Appl. Climatol., 115, 15-40, doi:10.1007/s00704-013-0860-x, 2014.

Taylor, P.: Intercomparison and Validation of Ocean-Atmosphere Energy Flux Fields - Final report of the Joint WCRP/SCOR Working Group on Air-Sea Fluxes, 325 pp., WCRP-112, WMO/TD-1036, 2000.

Villermaux, E. and Bossa, B.: Single-drop fragmentation determines size distribution of raindrops, Nat. Phys., 5, 697-702, doi:10.1038/nphys 1340, 2009.

Weller, R. A., Bradley, E. F., Edson, J. B., Fairall, C. W., Brooks, I., Yelland, M. J., and Pascal, R. W.: Sensors for physical fluxes at the sea surface: energy, heat, water, salt, Ocean Sci., 4, 247-263, doi:10.5194/os-4-247-2008, 2008.

WMO: Code Tables and Flag Tables Associated with BUFR/CREX Table B, available at: http://www.wmo.int/pages/prog/www/ WMOCodes/WMO306_vI2/LatestVERSION/WMO306_vI2_ BUFRCREX_CodeFlag_en.pdf (last access: 7 April 2016), 2015.

Yuter, S. E. and Parker, W. S.: Rainfall Measurement on Ship Revisited: The 1997 PACS TEPPS Cruise, J. Appl. Meteorol., 40, 1003-1018, doi:10.1175/15200450(2001)040<1003:RMOSRT>2.0.CO;2, 2001.

Yuter, S. E., Kingsmill, D. E., Nance, L. B., and Löffler-Mang, M.: Observations of Precipitation Size and Fall Speed Characteristics within Coexisting Rain and Wet Snow, J. Appl. Meteorol. Clim., 45, 1450-1464, doi:10.1175/JAM2406.1, 2006. 